\title{
Sexualidad, moral y cuentas falsas. Usos políticos de la edad de la primera relación sexual en Chile
}

\section{Sexuality, moral and false accounts. Political uses of the age of first intercourse in Chile}

\author{
Irma Palma \\ Universidad de Chile \\ ipalma@uchile.cl
}

\begin{abstract}
SÍNTESIS
Este artículo realiza una discusión metodológica y política sobre la medición de las edades de la primera relación sexual en las encuestas nacionales, analiza usos de instrumentos estadísticos, discute ciertas tesis e interpretaciones, y usos que hacen investigadores(as) del campo de estudios de género y de instituciones académicas católicas. Muestra que parte de la investigación de base estadística presenta errores en dicha medición, que conduce a conclusiones erradas; sin embargo, se le usa. Afirma que esto constituye un asunto al mismo tiempo técnico, académico y político; esto, en un contexto en que las encuestas han devenido en las sociedades contemporáneas en fundamento de la construcción de políticas, en instrumento de debates públicos o en referencia de la reflexividad institucional.
\end{abstract}

\begin{abstract}
This article makes a methodological and political discussion on the measurement of the age of first intercourse in national surveys, analyzes the use of the statistical instruments, discusses certain theses and interpretations, and uses of the research performed by researchers in the field of gender studies and by catholic academic institutions. It shows that part of the background research presents statistical errors in the measurement, which leads to erroneous conclusions. Nevertheless, it is used. It claims that this is a technical, academic and political issue in a context where surveys have become in contemporary societies in basis of policy making, in instruments of public debate, or as reference in institutional reflexivity.
\end{abstract}

Palabras claves:

Keywords: age of first intercourse, gender, sexuality autonomy 


\section{Introducción}

La producción de conocimiento científico en las ciencias sociales ha devenido en las sociedades contemporáneas en fundamento político-técnico de la construcción de políticas por parte de los estados, en instrumento obligado de debates públicos o en referencia de la reflexividad institucional respecto de un conjunto creciente y más complejo de fenómenos asociados a la sexualidad. En esto, la producción de la investigación de base estadística tiene un lugar notable.

Prolifera su tratamiento en encuestas en las sociedades occidentales contemporáneas. En Chile las realiza sobre todo el Estado, organismos no gubernamentales, agencias internacionales, medios de comunicación, agencias de estudios de opinión pública, y salvo excepciones, fuera de las universidades. Sus usos se multiplican. Las usan los decisores en el Estado para fundar técnicamente el diseño de sus políticas. Su uso se ha vuelto orgánico a una comprensión del diseño de la política pública y de la deliberación ciudadana, y del lugar de la ciencia en ello, donde los(as) investigadores(as) parecen aspirar a fundarla, no a informar la deliberación ciudadana (Fassin, 2005). Son utilizadas por la sociedad civil en la construcción de argumentos para la abogacía ante el Estado y debates públicos. Estas parecen producir un efecto de empoderamiento en los(as) investigadores(as) y activistas, pues permiten dialogar con el decisor en su terreno y en su lenguaje -el técnico. Inusitadamente, las usan instituciones religiosas en su argumentación, como sucedió en el debate de la ley de divorcio, en 2004. Los medios de comunicación han llegado a producirlas y financiarlas. Suelen presentarlas como evidencia, como si no tuviesen margen de interpretación -pura objetividad-; suelen producir un efecto de verdad sobre las audiencias, pues parecen mostrar a la sociedad en un solo dato.

Cualquiera sea su naturaleza, sea la sexualidad su objeto principal o no, y cualesquiera sus fines, encuestas situadas en campos diversos -política, salud, género, juventud, niñez y mujeres- indagan sobre prácticas, edades del sexo, uso de tecnología preventiva, parejas sexuales, abuso sexual contra niños/as, conocimientos, contextos sexuales, etc. Se exploran orientaciones normativas y también juicios políticos; incluso sobre las instituciones, como sucede respecto de la Iglesia Católica y del Estado. 
Como sostiene Michel Bozon (2009), las encuestas son al mismo tiempo, una producción intelectual, un emprendimiento social y político, y un producto cultural. Son una producción intelectual porque contribuyen a construir y legitimar un objeto científico, imponen definiciones, utilizan categorías, producen nuevos datos. Como otra investigación social, las encuestas se mueven en una tensión entre descubrir y construir en el proceso de observar los fenómenos que estudian. Las categorías conceptuales que permiten su comprensión también sirven a su construcción (Gagnon, 1991) Posiblemente, el caso del embarazo en la adolescencia resulte paradigmático para observar esta tensión. Son un emprendimiento social y político, pues una encuesta surge a partir de un fenómeno que se constituye en problema socialmente identificado y reconocido, al cual concurren actores institucionales -en alianza o en conflicto- que las impulsan, demandan, hacen, financian o utilizan. Finalmente, son un producto cultural, que actúa como guía de lectura de la realidad, como sucede cuando instituciones vinculadas a la promoción de la despenalización del aborto miden la evolución de las orientaciones normativas y juicios políticos en la sociedad chilena.

Este trabajo es un ejercicio propiamente metodológico y, al mismo tiempo, es una reflexión política sobre las encuestas. Aquí trato la edad de la primera relación sexual en la sociedad chilena, pero un sentido ésta opera como un analizador: pudo ser lo mismo interrogada la medición de otros fenómenos.

Este ejercicio se inspira en un interés personal que puede ser expresado en términos de una auto-observación y reflexividad disciplinaria que quisiera favorecer. Durante muchos años he planteado a estudiantes de pregrado, de magister y doctorado un par de preguntas sencillas. La primera: ¿a qué edad tienen la primera relación sexual los(as) los(as) jóvenes en el país? Hasta ahora nadie ha dicho: "No sé". En su gran mayoría indican edades comprendidas entre doce y quince años. Luego, digo: ¿cómo lo sabe? Sus fuentes son reportajes de prensa, algún pariente o sus pacientes. Luego, hago una segunda pregunta: ¿cuál es la prevalencia de la depresión en Chile? La enorme mayoría dice: "no sé". Luego, digo: ¿cómo lo sabe (o no)? Responden que no han leído un artículo científico. Y les hago unas últimas preguntas: ¿Por qué la primera pregunta no tiene una respuesta equivalente a la última? ¿Cómo interpretar el hecho que 
un(a) estudiante de doctorado responda fuera del campo de la ciencia a la primera pregunta, parezca bastarle una fuente prosaica y no se interroga por la racionalidad de su dato?

Se inspira también en una crítica sobre cierta investigación de base estadística que se lleva adelante en el país, y sobre todo en una preocupación en torno a sus usos. Existe una pequeña encuesta realizada en 1996-97 con solo 229 adolescentes de 11 a 17 años de enseñanza básica de tres escuelas de Temuco a las cuales asisten estudiantes pobres (Fernández et al., 2000), encuesta que resulta metodológicamente insostenible, que ha sido y sigue siendo sistemáticamente utilizada por ciertos(as) investigadores(as) para evidenciar lo que constituiría una tendencia acelerada hacia la precocidad sexual en las nuevas generaciones. En ese mismo momento otra encuesta se hacía en el país. Era la II Encuesta Nacional de Juventud, con 3.446 hombres y mujeres entre 15 y 29 años, y representatividad nacional. Mientras la primera concluía -sobre la base de solo de 26 estudiantes activos sexualmente- que la edad promedio es 12,83 $\pm 3,13$ años en las mujeres y 12,0 $\pm 2,27$ años en los hombres, la última encontraba edades medias de 18,6 en las mujeres y 16,1 años en los hombres. ¿Por qué esa remota encuesta pudo ser hecha del modo en que lo fue, pero sobre todo, por qué puede ser utilizada hasta hoy en la academia?

La edad de la primera relación sexual está presente al modo de un indicador en los discursos institucionales religiosos sobre la sexualidad y las generaciones, parte de una interpretación de la sexualidad contemporánea. En los discursos de las instituciones de salud -con el riesgo de una intensa medicalización de la sexualidad ${ }^{1}$. En los fundamentos de la abogacía sobre asuntos públicos de la salud y derechos sexuales y reproductivos. Está en la educación sexual, cuando se la demanda en razón de una supuesta precocidad, y en propuestas institucionales que intentan su postergación. Se encuentra en los medios cuando desnudan la sexualidad juvenil, que suelen usar el caso extremo y no el caso normal; la adolescente que tuvo sexo por primera vez a los doce años conmueve, la que lo hizo a los diecisiete no.

Busco hacerme cargo de una discusión metodológica y política sobre la medición de las edades de la primera relación sexual, analizo ciertos usos de instrumentos, discuto ciertas tesis e interpretaciones, 
y usos de la información producida. Busco hacerme cargo de una discusión metodológica y política sobre la medición de las edades de la primera relación sexual, analizo ciertos usos de instrumentos, discuto ciertas tesis e interpretaciones, y usos de la información producida. Aquí analizo las formas de la medición, así como los usos que hacen investigadores(as) de instituciones académicas católicas y del campo de estudios de género.

Parte importante de la investigación que se desarrolla en el país presenta errores en la medición de las edades, que conduce a conclusiones erradas; sin embargo, se le usa. Al mismo tiempo, aparentemente fundada, existe una producción de interpretaciones que no se someten a la crítica científica y política. Parece tratarse no solo de un error científico; se trata también de una cuestión al mismo tiempo técnica, académica y política.

Sugiero que sus usos podrían resultar paradojales para los estudios de género. Ha descendido la edad de la primera relación sexual -y desinstitucionalizado- en las mujeres y estabilizado en los hombres, y en el campo de estudios de género parece existir una tensión del orden de las categorías para una interpretación. La academia católica lo interpreta. Lo define como prematurez e intensifica una interpretación sobre la transformación en las edades sexo de las nuevas generaciones como un indicador de liberalización extrema y desregulación normativa en el orden de la sexualidad, y una inédita y estructural proximidad al riesgo.

Sin necesariamente proponérselo, y si los(as) investigadores(as) del campo de los estudios de género solo intentasen relevar el hecho de una creciente proporción de jóvenes sexualmente activos(as), dado que no interpretan el sentido del cambio $-\mathrm{y}$ siempre aparece cerca del riesgo-, dicha tendencia aparece por sí misma dramática. A la precocidad de la academia católica no se le opone nada en el plano de las edades, sino que se le replica en el de su abstencionismo. Cabe la posibilidad que sin proponérselo, unos(as) y otros(as) se encuentren en el riesgo, solo que en sus dos versiones, la del riesgo moral y la del riesgo sanitario. Sugiero interrogarse por las mujeres jóvenes y la autonomización de la sexualidad respecto de la conyugalidad y la reproducción, y 
ponerla en conexión con una apertura a la autonomía sexual; esto, sin embargo, en el contexto de una transformación socialmente diferenciada de la estructuración social de los calendarios y la reconfiguración de las trayectorias sexuales y no sexuales.

Parece contradictorio el hecho que en el campo de estudios de género se haga uso de las encuestas cuando se trata de la política, y aun cuando se hace una sistemática crítica a los métodos cuantitativos -se discute cuestiones epistemológicas, metodológicas y tecnológicas-, se lo hace sin someter a la crítica la investigación real, aquella que se hace en el país, sus diseños, objetos, resultados e interpretaciones. No hacerlo tiene consecuencias políticas. En esto participamos investigadores(as). Por ello, sostengo que la capacidad de auto observar las propias observaciones permitirá transparentar las mediciones y categorías en el campo de los estudios de género, y de esta forma fomentar el trabajo científico promoviendo capacidades reflexivas en las ciencias sociales en el país. Cuando analizo y critico lo que hacen los(as) investigadores(as), lo hago también respecto de mí misma.

\section{Parte: La medición}

Determinar las edades en que acontece la primera relación sexual resulta complejo, pues se trata propiamente de investigar la estructura de las edades de entrada en la sexualidad activa en una sociedad determinada, en una generación, en una población o grupo social ${ }^{2}$. Cuando se mide en una cohorte muy joven, como ocurre habitualmente en la investigación social, no se captura propiamente la estructura de las edades del sexo. Cuando no se establece una distancia temporal aparece como un fenómeno muy temprano en la vida. Esto, porque en cohortes etarias muy jóvenes suele hallarse una proporción baja de sujetos que ya han tenido sexo, y la investigación que mide las edades del sexo en una población muy joven tiene que hacerse cargo de los individuos que no han tenido aún.

A la investigación siguiente puede hacérsele una crítica metodológica (Fétis et al., 2008). Fue realizada en 2008, también en Temuco, con 698 estudiantes de enseñanza media, y su edad promedio 
de $16,2 \pm 1,4$ años, $53,8 \%$ son mujeres, $50,4 \%$ van a establecimientos particulares subvencionados, $34,1 \%$ a municipales y $15,5 \%$ a particulares pagados. En dicha investigación, solo 17,04\% había tenido ya una primera relación sexual, y sobre la base de esa proporción de sujetos las edades medias encontradas son 15,5 años en las mujeres y 14,3 años en los varones. Esto, cuando $82,96 \%$ aún no tenía sexo. Una consecuencia grave de un error de este tipo se ubica en la interpretación de sus hallazgos. En la interpretación la media deviene mayoría. (De Irala et al., 2011) Dado que además del error metodológico de base, los investigadores al comunicarlos no informan tampoco la proporción de sujetos que han tenido sexo, quienes los leen creen que la mayoría lo ha hecho a esa edad. Este es un efecto aparente, pues puede ser exactamente lo contrario, tal como sucede en ambas investigaciones.

\section{Usos de la media y la mediana}

La proporción de sujetos que ha tenido una primera relación sexual es progresiva en las edades. Esto puede ser observado aquí usando la VI Encuesta Nacional de Juventud, de 2009. (Tabla 1)

Tabla 1: Proporción de sujetos que tienen la primera relación sexual a cada edad específica y edades media y mediana de primera relación sexual según sexo y cohortes etarias. (15 y 29 años)

\begin{tabular}{|c|c|c|c|c|c|c|}
\hline & Año de nacimiento & 1992-94 & 1990-91 & $1985-89$ & $1980-84$ & 1980-1994 \\
\hline & Edad 1aㅡ RS /Edad actual & $\begin{array}{l}15-17 \\
\text { años }\end{array}$ & $\begin{array}{l}18-19 \\
\text { años }\end{array}$ & $\begin{array}{l}20-24 \\
\text { años }\end{array}$ & $\begin{array}{l}25-29 \\
\text { años }\end{array}$ & $\begin{array}{l}15-29 \\
\text { años }\end{array}$ \\
\hline \multirow{10}{*}{ Hombre } & 13 o menos & 5,5 & 6,6 & 5,2 & 7,2 & 6 \\
\hline & 14 & 7 & 7,4 & 8,5 & 9,4 & 8,3 \\
\hline & 15 & 14,1 & 12,6 & 13,8 & 9,8 & 11,6 \\
\hline & 16 & 8,7 & 17,5 & 14,2 & 13 & 13,7 \\
\hline & 17 & 1,7 & 19,2 & 18,8 & 17,7 & 17,7 \\
\hline & 18 o más & - & 8,3 & 27,2 & 37,2 & 31 \\
\hline & Activos sexualmente & 37,1 & 71,6 & 87,7 & 94,4 & 88,2 \\
\hline & No activos sexualmente & 62,9 & 28,4 & 12,3 & 5,6 & 11,8 \\
\hline & Edad media & 14.8 & 15.8 & 16.5 & 16.9 & 16.4 \\
\hline & Edad mediana & 15 & 17.3 & 17.4 & 17.7 & 17.6 \\
\hline
\end{tabular}




\begin{tabular}{rlrrrrr}
\hline \multirow{4}{*}{ Mujer } & 13 o menos & 2,5 & 1,4 & 2,6 & 2,6 & 2,3 \\
& 14 & 6,7 & 4,8 & 5,6 & 6,6 & 5,6 \\
& 15 & 10 & 13 & 11,8 & 7,4 & 9,9 \\
& 16 & 10,2 & 13,6 & 11,9 & 9,9 & 11 \\
& 17 & 3,9 & 22,1 & 16,5 & 19 & 17,8 \\
& & - & 11,9 & 36,9 & 50,2 & 39,9 \\
& Activos sexualmente & 33,2 & 66,9 & 85,2 & 95,7 & 86,6 \\
No activos sexualmente & 66,8 & 33,1 & 14,8 & 4,3 & 13,4 \\
& Edad media & 15.2 & 16.3 & 17.1 & 17.8 & 17.1 \\
& Edad mediana & 15 & 17,8 & 18.0 & 18,2 & 18.1 \\
\hline
\end{tabular}

Fuente: Elaboración propia en base a la VI Encuesta Nacional de Juventud, INJUV, 2009.

La media y la mediana ofrecen mejor una medición del pasado que del presente, de cohortes mayores que de aquellas en que los sujetos están proceso de entrada en la sexualidad activa. La media hace referencia a una variable que considera valores -en este caso, edad de la primera relación sexual de cada sujeto que ya la ha tenido- y no considera a los que no asumen un valor -que no la han tenido aún- o no es aplicable. Corresponde al valor promedio de la variable, es decir, a la edad promedio del segmento iniciado, y presenta la desventaja que es afectada por valores extremos: algunos individuos que se inician a edades tempranas o tardías. Cuando se utiliza la media se excluye a los no iniciados, entonces se mide sólo a parte de la población que ha tenido la conducta. La media tiene una relación particular con los rangos de edad de los sujetos sometidos a investigación. Cuando se utiliza la media en una población de adolescentes (10 a 19 años de edad), como es una población en proceso de entrada a la sexualidad activa, y es presumible que una proporción importante no haya iniciado sus prácticas sexuales, el estadístico arroja un valor menor (más bajo mientras más pequeño sea el tamaño del grupo ya iniciado sexualmente) al que se podría obtener unos años posteriores con el mismo grupo. Por ello, mientras menor sea la proporción de sujetos iniciados sexualmente, más difícil resulta que la media exprese al conjunto de la población estudiada. Por esto, cuando se usa la media se tiene que mostrar la proporción de sujetos que ha tenido una primera relación sexual. 
La mediana expresa la edad en que el $50 \%$ de los individuos tuvo una primera relación sexual a menor edad y el otro $50 \%$ lo hizo a una edad mayor. Cuando se utiliza la mediana de la edad de iniciación sexual el procedimiento se basa en que la mayoría de los entrevistados ya la han tenido y es un hecho pasado, es decir, la edad del evento ya está establecida. Cuando se aplica la mediana a la edad de iniciación de una población de adolescentes, y es presumible que una gran parte de los sujetos con edades menores a la mediana no hayan iniciado sus prácticas sexuales, el procedimiento incluye los no iniciados, para ello se traslada los no iniciados al final de la distribución de valores, entonces, el estadístico arroja un valor superior al que se podría obtener unos años posteriores con el mismo grupo. Cuando la mediana de edad de iniciación sexual coincide en términos generales con las edades de la población estudiada, no hay dificultad en presumir que los sujetos que no lo han hecho lo harán después. Así ocurre en la encuesta de la Comisión Nacional de Sida (CONASIDA/ANRS, 1998), donde la edad de la población estudiada es de 18-69 años, y las edades medianas de iniciación son menores que la edad mínima de la población estudiada (18 años): en el grupo de 18-19 años de hombres es 17.3 años. En ellos se puede calcular porque la edad mediana es inferior al límite bajo de la edad de la población estudiada (72.4\% lo ha vivido en el caso 18-19 años) Por el contrario, en el caso de las mujeres, la edad mediana se puede sólo calcular para el grupo de 19 años, porque en el grupo 18-19 años, $53 \%$ no lo ha vivido) a los 19 años la edad mediana es de 18.7 años. Por ello, sugiero que puede ser inadecuado calcular una mediana de edad en una cohorte en que la cual la mitad de los sujetos con la edad mínima todavía no han vivido el evento. Si se busca conocer la edad en que un grupo poblacional tiene la primera relación es preferible usar cohortes un poco mayores dentro del mismo grupo, de modo de asegurar que un mayor porcentaje de sujetos ya la haya tenido.

Se ha sugerido, y lo comparto, que cuando se trabaja con cohortes adolescentes es mejor el uso de la proporción de sujetos que tienen una primera relación sexual en unas edades determinadas. (De Irala et al., 2011) Cuando se busca saber cuán tempranas o tardías pueden ser y cuántos lo hacen en esas edades en un grupo determinado, sirve usar las frecuencias específicas de las edades de primera relación 
sexual. Si se usan las frecuencias de sujetos que se inician en cada edad específica se tiene con precisión la distribución, desde los más precoces a los más tardíos. Si se busca saber la proporción total de sujetos que en una población se encuentran sexualmente activos en cada edad específica, puede usarse una medición que incorpore las proporciones de las edades anteriores.

\section{Parte: Los análisis}

En Chile se realizó a fines de la década de los noventa la primera y única encuesta nacional sobre sexualidad, que fue desarrollada por CONASIDA con la colaboración de la Agence Nationale de Recherche sur le SIDA, de Francia en 19983. Antes, el régimen militar se negó a continuar participando en la Encuesta Mundial de Fecundidad (DHS), que en parte la hubiese considerado. Otras encuestas que realiza el Estado en forma sistemática permiten, no obstante, estudiar ciertos elementos. Está la serie de la Encuesta Nacional de Juventud, que hace desde 1994 el Instituto Nacional de la Juventud, que tiene ya seis mediciones periódicas desde 1994 a 2009². Está también la Encuesta Nacional de Salud, que hace el Ministerio de Salud (su primera versión fue hecha en 2003 y la segunda y última versión fue hecha en 2009-2010)5. Está también la Encuesta Nacional de Calidad de Vida y Salud, que hace el Ministerio de Salud y el Instituto Nacional de Estadísticas (su primera versión fue hecha en 2000, la siguiente en 2006 y la tercera se encuentra en desarrollo) ${ }^{6}$. Finalmente, está la Encuesta Nacional de Salud Escolar, que trabaja con estudiantes de $7^{\circ}$ y $8^{\circ}$ de enseñanza básica, y $1^{\circ}$ año de enseñanza media de todos los colegios municipalizados y particulares subvencionados de las regiones I, V , XIII y VIII'.

\section{Planes de análisis}

La primera relación sexual es un evento en la vida de un sujeto que se inscribe en una trayectoria sexual, y está conectada con las edades en que suceden otros eventos; esto, porque se sitúan en una estructuración social de calendarios que hacen no sólo a la sexualidad a la conyugalidad ${ }^{8} \mathrm{o}$ a la reproducción, sino también a eventos no sexuales, edades para el estudio, el ingreso al trabajo, etc. Son recorridos biográficos individuales. Trayectoria es un concepto que 
permite interpretar mejor eventos individuales que no están desconectados unos de otros en el conjunto de la vida de un sujeto y que, a su vez, son comunes a ciertos individuos y grupos, y al mismo tiempo, disímiles a otros. Esto último refiere a la estructuración social de los calendarios -tiempos, momentos, distancias entre eventos, secuencias, sincronías, desfases, etc.- del sexo, la conyugalidad o la reproducción, a su organización en un grupo o sociedad, y su inscripción en la estructura social y en las relaciones sociales.

\section{Edades y generaciones, género y clase}

El concepto de "generación" remite al problema de la elaboración de diferencias entre los miembros de diferentes cohortes de edad, definidos previamente, cuando se modifican las condiciones materiales y socioculturales de existencia y de reproducción de eso grupos. El concepto de generación no remite a la constitución de grupos concretos, aunque pueda darles fundamento. No se puede hablar de una generación más que en la medida en que quienes entren simultáneamente en la vida participen potencialmente en acontecimientos y experiencias que crean lazos. Sólo un mismo cuadro de vida histórico-social permite que la situación definida por el nacimiento en el tiempo cronológico se convierta en una situación sociológicamente pertinente (Mannheim, 1990).

Se trata, por tanto, de la introducción histórica y social de rupturas que modifican las condiciones de la actividad sexual. Sin embargo, ¿cómo se delimita las generaciones? La demarcación de límites generacionales obedecerá a las categorías que los investigadores utilicen y consideren relevantes y de cómo se definan las condiciones sociales y materiales de un grupo social o sexual determinado. Tales condiciones constituyen los contextos de ejercicio de la sexualidad. Se trata de elementos que modifican las condiciones de vida de los individuos, como el incremento de niveles educacionales o la participación de las mujeres en el mercado del trabajo -que aumentan su autonomía con respecto a los hombres-, los calendarios y los modos de entrada en la vida conyugal, normatividad institucional, contexto sanitario y epidemiológico en un periodo determinado, condiciones de la contracepción, etc. ${ }^{9}$ (Mossuz-Lavau, 1991). Cuando se diseñan cohortes y no se considera la categoría de generación en los planes de análisis puede producirse un efecto aparente, pero erróneo de 
una inflexión drástica en la evolución de las edades de entrada en la sexualidad activa.

En Chile, la edad de la primera relación sexual en las mujeres muestra una tendencia sistemática al descenso a lo largo de las generaciones durante el siglo veinte, sin ninguna inflexión dramática. (Gráfico 1) Los hombres, en tanto, han tenido históricamente edades más tempranas, y en las últimas generaciones una cierta estabilización en las edades. (Gráfico 2)

Gráfico 1: Evolución de las edades medianas de la primera relación sexual en generaciones desde 1930 a 1991 en mujeres, según nivel educacional.

Fuente: Elaboración propia en base a Estudio Nacional de Comportamiento Sexual (CONASIDA/ANRS, 1998) para cohortes 1930 a 1979 y VI Encuesta Nacional de Juventud (INJUV, 2009) para cohortes 1980 y 1991.

Gráfico 2: Evolución de las edades medianas de la primera relación sexual en generaciones desde 1930 a 1991 en hombres, según nivel educacional.

Fuente: Elaboración propia en base a Estudio Nacional de Comportamiento Sexual (CONASIDA/ANRS) para cohortes 1930 a 1979 y VI Encuesta Nacional de Juventud (INJUV, 2009) para cohortes 1980 y 1991.

Suele decirse que en la sociedad chilena la introducción de la tecnología anticonceptiva a mediados de la década de 1960 habría modificado las condiciones de entrada en la sexualidad activa en las generaciones contemporáneas, que se habría reducido drásticamente la edad de la primera relación sexual entre las mujeres, y que el movimiento hippie la habría puesto fuera del matrimonio. Sin embargo, es notable el hecho que no se encuentra una inflexión sustantiva en esas generaciones. En realidad en el momento en que se iniciaba la política de planificación familiar se encontraba ya en marcha una transformación de las trayectorias sexuales femeninas. La generación que iniciaba la actividad sexual contemporáneamente al origen 
de la política de planificación familiar -esto es, los nacidos a partir de 1950- hizo un uso muy escaso de la tecnología contraceptiva. En su origen dicha política no acopló con este proceso de constitución de una inédita sexualidad juvenil, definida como una separación temporal de los calendarios de la sexualidad, la reproducción y la conyugalidad. Esta no se dirigió a las mujeres jóvenes solteras y nuligestas, ni se orientó al retardo de la primera maternidad. La política de planificación familiar tendría que haberse extendido a los grupos de mujeres jóvenes en el periodo de dictadura (1973-1989) y tuvo, por el contrario, una contracción en su cobertura ${ }^{10}$.

Gráfico 3: Evolución del uso de tecnología preventiva en la primera relación sexual de las generaciones nacidas entre 1950 a 1991, según sexo.

Fuente: Elaboración propia en base a Estudio Nacional de Comportamiento Sexual (CONASIDA/ANRS) para cohortes 1950 a 1974, V Encuesta Nacional de Juventud (INJUV, 2006) para cohorte 1977 a 1979 y VI Encuesta Nacional de Juventud (INJUV, 2009) para cohortes 1980 y 1991.

A su vez, se ha producido una transformación de los contextos en que se ésta realiza. Se trata de una des-institucionalización de la primera pareja sexual en las mujeres y al mismo tiempo que en los hombres (que nunca lo hicieron en el matrimonio) crece el contexto relacional (más que antes lo hacen en el pololeo). Durante el siglo veinte las mujeres sustituyen al marido por el pololo. Esto es progresivo en el tiempo. En la encuesta CONASIDA/ANRS (1998) en torno a $67 \%$ de mujeres nacidas en la década del treinta tuvieron como primera pareja sexual al esposo, y en quienes nacieron en la década de los ochenta se ubicaba en torno al 1\%. Los hombres, en cambio, lo hicieron siempre a gran distancia de la conyugalidad (Palma, 2006).

\section{Edades y trayectorias, género y clase}

En realidad no es la edad de la entrada en la sexualidad activa, sino el recorrido biográfico lo que importa. Una misma edad en Bangladesh, Japón, Suecia o México no son lo mismo. En la primera de estas sociedades, entre las mujeres pubertad, iniciación sexual, con- 
yugalidad y fertilidad son eventos temporalmente muy próximos; en la segunda, todos estos eventos son tardíos; en la tercera, ésta es temprana y los otros dos eventos muy tardíos; y en la última, en contexto tradicional, fue mucho más tardía que en los hombres y conectada a la conyugalidad y la fertilidad. (Bajos et al. 2004; Bozon, 2003; Bozon y Hertrich, 2001; Bozon y Kontula, 1997). Puede haber una misma edad de la primera relación y, en cambio, muy diversas trayectorias. A su vez, pueden operarse transformaciones más importantes en otros de estos eventos. También sucede que la postergación de uno de estos eventos puede conllevar la de otros, como sucede en países africanos en los cuales tradicionalmente las mujeres entraron en la conyugalidad después de la pubertad, y las políticas educacionales y nuevas tendencias culturales han ido retardándolo, y de ese modo, la reproducción, y con ello la primera relación sexual se retrasaría.

La primera relación sexual es un evento en la vida de un sujeto que se inscribe en una trayectoria sexual, y está conectada con las edades en que suceden otros eventos; esto, porque se sitúan en una estructuración social de calendarios que hacen no sólo a la sexualidad a la conyugalidad o a la reproducción, sino también a eventos no sexuales, edades para el estudio, el trabajo, etc. Son eventos individuales que no están desconectados unos de otros en el conjunto de la vida de un sujeto y que, a su vez, son comunes a ciertos individuos y grupos, y al mismo tiempo, disímiles a otros. Esto último refiere a la estructuración social de los calendarios -tiempos, momentos, distancias entre eventos, secuencias, sincronías, desfases, etc. del sexo, la conyugalidad o la reproducción-, a su organización en un grupo o sociedad, y su inscripción en la estructura social y en las relaciones sociales.

En la sociedad chilena las trayectorias sexuales no son socialmente homogéneas. En general, las encuestas no han incorporado la categoría de trayectoria en sus instrumentos y análisis cuando estudian la sexualidad. La serie de encuestas nacionales de juventud dificulta analizar las trayectorias, entre otras cosas, porque no incluye algunos de estos eventos ni las edades en que ocurrieron algunos de los que incluye. ${ }^{11}$ No obstante, si bien no se consignan todos los eventos ni los momentos en que ocurrieron, podemos mediante otra forma indagarlas. Podemos hacerlo mediante la proporción de sujetos que 
en una cohorte se encuentran sexualmente activos, están unidos ${ }^{12}, \mathrm{y}$ tienen hijos/as. Intento mostrar la importancia de analizar las trayectorias en la investigación social, y lo hago mediante las trayectorias reproductivas y conyugales femeninas y sus niveles educacionales ${ }^{13}$.

En las trayectorias biográficas las jóvenes presentan una importante diferenciación social de las edades de la fertilidad en el curso de sus trayectorias reproductivas. Las mujeres con niveles de educación superior presentan una fecundidad más tardía, escasamente iniciada en la adolescencia, y altamente postergada hasta pasado el periodo de juventud. En sentido inverso, aquellas con niveles de educación básica o media incompleta presentan una fecundidad más temprana, en una alta proporción en la adolescencia, que no se extiende por todo el periodo, sino que tiende a concluir antes de los 25 años. En la VI Encuesta Nacional de Juventud (2009), en el grupo entre los 25 y 29 años de edad, solo 15,2\% de las primeras tuvo hijos antes de los 20 años, y de las últimas, 62,9\%; y 50,4\% de las primeras no ha tenido hijos, y de las últimas, solo 4,6\%. (Palma, 2012) A su vez, las trayectorias conyugales (que entendemos genéricamente como uniones, sean maritales o cohabitación) presentan una importante diferenciación social. Aquellas mujeres con niveles de educación superior presentan más bajos niveles y más tardías las edades, y en sentido inverso, aquellas con niveles de educación básica o media incompleta presentan más altos niveles y más tempranas las edades de uniones (en el grupo entre 25 y 29 años, 63,8\% y 21,9\%, respectivamente, permanecen solteras) (Palma, 2012).

En los grupos de ingresos altos en su gran mayoría las jóvenes acceden a la educación superior, y se encuentran en condiciones de ingresar al mundo laboral en posiciones más ventajosas. En sus trayectorias biográficas postergan la conyugalidad y la fertilidad hasta la adultez, aunque no necesariamente la entrada en la sexualidad activa. Sus trayectorias reproductivas se encuentran poco interferidas por la existencia del embarazo no previsto y más preventivamente más protegidas. En cambio, las mujeres jóvenes en los grupos socioeconómicos de más bajos ingresos pueden cruzar barreras educacionales intermedias, y muy difícilmente la de la educación superior, y se encuentran en condiciones de ingresar al mundo laboral precariamente. En sus trayectorias biográficas tienden a postergan menos la conyugalidad, aunque sí procuran hacerlo con 
su fertilidad -como se colige del hecho que una proporción importante declara haber experimentado un embarazo no previsto. Sus trayectorias reproductivas se encuentran interferidas por la existencia del embarazo no previsto en razón de un muy bajo nivel de uso de tecnología preventiva, y es probable que comiencen sus prácticas preventivas justamente después de esta experiencia (Palma, 2012).

\section{Parte: Los usos}

En 2005 en un artículo publicado en la Revista Médica de Chile por investigadores de la Pontificia Universidad Católica (PUC) (Vigil et al, 2005), se utiliza como referencia para un análisis de las edades de entrada en la sexualidad activa un conjunto de estudios realizados en $1988^{14}$, en 1996-1997 ${ }^{15}$ y la pequeña investigación realizada en 1996-1997 en Temuco. (Fernández et al, 2000) ¿Cómo explicar diferencias tan grandes en las edades (12 y 16 entre los hombres; 14 y 17,9 entre las mujeres)? ¿Por qué los investigadores operan sólo con las edades más bajas para afirmar cuáles son las edades? Las primeras de estas edades indicarían que sucede en el mismo momento de la maduración sexual.

El inicio de la actividad sexual en los jóvenes chilenos es a los 12 años para los hombres y 14 años para las mujeres. Estudios efectuados en Chile entre los años 1992 y 1995, mostraron que (...) que la edad de inicio de la actividad sexual, entendida ésta como la primera relación sexual, era en promedio, entre los 14,4 y 16 años en los hombres y entre los 14,8 y 17,9 años en las mujeres. $(1,5)$ Trabajos más recientes indican un inicio más precoz de la actividad sexual siendo de 12 años para los hombres y 12,8 años para las mujeres (6) (1174).

Cuatro años más tarde, en 2009, la misma autora y colaboradores (Vigil et al., 2009), usando esta vez como base estudios realizados en $2006^{16}$ y $2007^{17}$ (González et al., 2007) y dejando fuera la pequeña investigación de Temuco, hallan unas edades más próximas a las mayores a las anteriores. Siguiendo a los investigadores de la PUC en sus dos comunicaciones -2005 y 2009- la tendencia de las edades en el tiempo sería justamente en sentido contrario al sugerido. 
Los estudios más recientes muestran que el inicio de la actividad sexual -entendido esto como la primera relación sexual genitalen los jóvenes chilenos es a los 16 años para los hombres y 17 años para las mujeres según la Quinta Encuesta del Instituto Nacional de la Juventud (INJUV) (2); mientras que otros estudios reportan como edad de iniciación sexual a los 15,7 y 15,5 años para varones y mujeres, respectivamente (3) (198).

En un artículo de 2008 (León et al., 2008), investigadores de la Universidad de Los Andes utilizan tres estudios realizados en el país para concluir en la existencia de una tendencia al descenso en las edades del $\operatorname{sexo}^{18}$. Incorporan, por cierto, la pequeña investigación de Temuco 1996-1997.

Según la Cuarta Encuesta Nacional de la Juventud, en Chile, al año 2003, el 35,2 de los adolescentes y el 84,5 de los jóvenes hasta los 24 años de edad se encontraban sexualmente activos. (3) Al año 2003 se estimaba que la edad promedio de inicio de las relaciones sexuales era alrededor de los 17 años, sin embargo en trabajos más recientes al respecto, se ha visto una tendencia a una mayor precocidad. Como ejemplo de lo anterior es posible citar el estudio realizado por González T et al el año 2005 en donde se vio que la edad promedio de inicio de la actividad sexual era de 15,6 años. $(3,6)$ Sin embargo un trabajo realizado el año $2000^{19}$ en Temuco, reveló una mayor precocidad del inicio de la actividad sexual siendo de 12 años para los hombres y 12,8 años para las mujeres (7) (43).

Un análisis hecho en la Universidad de la Santísima Concepción afirma usa datos que evidenciaría un descenso acelerado. ${ }^{20}$ Usa la Cuarta Encuesta Nacional de la Juventud (2003), es presumible que usa también el estudio que trabaja con 198 adolescentes de una comuna de la Región Metropolitana (González et al., 2005) y el estudio realizado en 1996-1997, en Temuco. (Fernández et al., 2000).

Según la Cuarta Encuesta Nacional de la Juventud, en Chile, al año 2003, el 35,2 de los adolescentes y el 84,5 de los jóvenes hasta los 24 años de edad se encontraban sexualmente activos. Además se estimaba que la edad promedio de inicio de las relaciones sexuales era alrededor de los 17 años, sin embargo trabajos posteriores indicaban promedios de 15,6 y hasta 12 años, siendo variables según 
región y niveles socioeconómicos.

En 2012, la Universidad San Sebastián hace también un análisis sobre las edades de entrada en la sexualidad activa. Usa, en primer lugar, al Instituto Nacional de Estadísticas, en segundo lugar refiere al artículo de investigadores de la PUC de 2005, mencionado anteriormente, en tercer lugar, usa un estudio de 2008, que no fue encontrado; en cuarto lugar, usa la Sexta encuesta nacional de juventud, de 2009. Sus autores afirman que los hombres y mujeres en las nuevas generaciones tienen la primera relación sexual muy tempranamente y tienden a la prematuridad, aunque sus datos usados varían entre 13,9 a 16,35 años en los hombres, y 15,5 a 17,7 años en las mujeres.

Es así que las estadísticas nos muestran que nuestros adolescentes están comenzando su actividad sexual muy tempranamente, en promedio 16,2 años los hombres y 17,7 las mujeres (INE, 2004). Un estudio realizado por la Pontificia Universidad Católica de Chile encontró mayor prematuridad al respecto, donde la edad de inicio de la primera relación sexual para las mujeres había sido en promedio 15,5 años y de 13,9 años para los hombres (Vigil P. y col 2005). Este último estudio concuerda plenamente con el estudio hecho por la Escuela de Enfermería de la Universidad San Sebastián en conjunto con el Servicio de Salud Concepción en junio del 2008, que muestra que nuestros escolares, en la provincia de Concepción están iniciando su actividad sexual a los 14 años +/- 1,3 años. La última encuesta INJUV (2010) da cuenta del inicio de la actividad sexual en los jóvenes chilenos, en promedio, a los 16,72 años (16,35 años para los hombres y 17,1 años para las mujeres)... (4).

Habría que interrogarse por la selección de los estudios. En el país es propiamente la serie de encuestas nacionales de juventud el instrumento de base para medir las edades de entrada en la sexualidad activa en las generaciones nacidas desde 1970 en adelante. Su uso aquí resulta extraño. A veces se le cita y no se le usa en la interpretación. Sugiero que esto es así porque sus edades son más elevadas y su tendencia es más moderada. Usan datos y casos extremos; por eso las pequeñas investigaciones mencionadas son sistemáticamente utilizadas en razón de que serían las edades más bajas producidas por la investigación social en el país. Sus análisis tratan sobre dos ca- 
tegorías, la de tendencia (la generación) y de precocidad (el sentido del cambio), y suelen intentar mostrar unas tendencias aceleradas hacia la precocidad con una drástica inflexión en las generaciones más recientes. Usan datos extremadamente heterogéneos, y no informan sobre sus universos y poblaciones (de modo de saber de qué sujetos se trata), su localización, y su representatividad. Las cohortes etarias estudiadas, el momento en que se hizo. A veces, incluso, carecen de las referencias bibliográficas obligatorias. Tratan las encuestas como si fuesen equivalentes, y por ello, comparables, lo que conduce a conclusiones erradas. No diferencian resultados locales de nacionales (ni siquiera con datos regionales y cohortes similares), operan con una encuesta con 229 sujetos como si fuese lo mismo que otra con 7500. El tratamiento de la sucesión temporal es problemático, cuestión fundamental cuando se estudian tendencias o evoluciones. Algunas de estas universidades usan datos que tienen una sucesión temporal (1997, 2003 y 2005), e invierten su orden. En apariencia, desde unas edades más elevadas (2003), se transita a unas más bajas (2005) y se llega a unas muy bajas (1997); esto, mediante una secuencia temporal que en el camino fue invertida.

Los(as) investigadores(as) del campo de estudios de género producen menos análisis sobre las edades de entrada en la sexualidad activa a partir de las encuestas que se realizan, publican menos artículos, hacen menos uso de la investigación de base estadística, y lo hacen fundamentalmente en documentos destinados a la política pública, a debates públicos y a comunicaciones en los medios.

En un artículo publicado en 2006 en la revista electrónica del Centro Latinoamericano en Sexualidad y Derechos Humanos, su autora afirma $^{21}$ :

Los comportamientos y prácticas sexuales de los jóvenes y adolescentes han sufrido variaciones importantes: la edad de iniciación sexual se ha reducido (66\% de los varones y $60 \%$ de las mujeres aproximadamente la inician entre los $15 \mathrm{y}$ los 18 años)...22.

Las proporciones encontradas parecer elevadas a su autora, sin embargo, no lo son, pues la distribución no es homogénea en las edades cubiertas en este rango etario, es decir son muchos más los que se encuentran próximos a los 18 que a los 15 años. 
El Proyecto de Ley Marco sobre Derechos Sexuales y Reproductivos presentado al parlamento en 2001, en su extenso diagnóstico sobre el conjunto de materias que constituyen su objeto sostiene ${ }^{23}$ :

\begin{abstract}
Según la Segunda Encuesta Nacional de la Juventud 1997, la gran mayoría de juventud entre 15 y 29 años declara haber tenido relaciones sexuales en alguna oportunidad. La iniciación en las relaciones sexuales tiende a concentrarse entre los 15 y 20 años apreciándose actualmente una iniciación más temprana en las mujeres que en 1994.
\end{abstract}

Cuando se dice que la edad de la primera relación sexual se ubica entre 15 y 20 años en verdad no se dice nada sobre las nuevas generaciones, pues casi todas generaciones del siglo veinte están incluidas en ese rango etario. Tampoco dicho dato permite concluir que existe un descenso en las edades. En 1994 como en 1997 o 2009, las edades encontradas se sitúan en ese amplio rango de 15 a 20 años.

Usando una errada asociación entre edad y uso de tecnología preventiva, según la cual baja edad y bajo uso serían inseparables, la academia católica encuentra allí un fundamento basal para la producción de un discurso sobre el riesgo moral. Más allá de ésta, ha dominado entre los(as) investigadores(as) la idea que la edad está obviamente asociada a un bajo uso de tecnología preventiva y, en consecuencia, a la exposición a riesgos sexuales y reproductivos el embarazo en la adolescencia, el VIH e ITS. El riesgo ha devenido estratégico. Por ello, el riesgo debe ser rigurosamente situado. Cuando el análisis sobre la asociación considera sólo edad y uso de tecnología preventiva, en la sociedad chilena se produce un efecto aparente de una correlación directa (a menor edad, menor uso), sin embargo, disimula el efecto real de la mediación de clase social, pues cualesquiera sean las edades, el uso se encuentra sistemáticamente más asociado a esta última que a la primera. En la VI Encuesta Nacional de Juventud (2009), cuando las mujeres tienen la primera relación sexual con 17 años o antes, 37,6\% usa tecnología preventiva, y cuando se produce a partir de los 20 años, 62,8\%. Sin embargo, si la edad fuese una variable que explica por sí sola esto, tendría que encontrarse mayores diferencias entre las edades que entre las clases sociales. Sucede que cuando una mujer con nivel de educación su- 
perior tiene la primera relación sexual con 17 años o antes, la usa en mayor proporción (55,0\%) que otra con educación básica o media incompleta que lo hizo a esa misma edad (24,9\%), y es extraordinariamente notable el hecho que cuando la primera la tiene a los 17 años o antes, usa tecnología preventiva en mayor proporción (55,0\%) que la última cuando lo hace a partir de los 20 años (38,1\%) (Palma, 2012).

Estas instituciones producen un discurso público y una propuesta a la política pública de abstinencia o la postergación de la edad de la primera relación sexual. La Pontificia Universidad Católica propone: "retrasar la iniciación de la actividad sexual"24. La Universidad Católica de la Santísima Concepción "fomenta la abstinencia sexual como una forma de decisión libre y responsable, a través de la virtud de la castidad, el don de la virginidad ${ }^{25}$. La tesis de la postergación necesita de la precocidad sexual para fundarse científicamente. La abstinencia católica deviene en método, y el método ha de devenir práctica. Y así es exactamente como se plantea el asunto por investigadores de la Universidad Católica (Vigil et al., 2005). Pero, en el curso del tiempo y de las generaciones se eleva el uso de condón y se reduce hasta su extinción la abstinencia. Ambas prácticas expresan tendencias culturales, una en ascenso (la protección devino exigencia) y la otra en declinación (se desdramatiza el sexo antes de la conyugalidad). En contexto de una creciente, aunque insuficiente instalación de la tecnología preventiva, la academia católica arriba al Estado, y le crítica en cuanto institución normativa, y denuncia una real o potencial política de derechos, pues otorgaría al sujeto joven un exceso de autonomía, y por ello, de contribuir a profundizar la tendencia a la precocidad.

La academia católica le usa como un indicador de tendencias profundas hacia una liberalización extrema y desregulación normativa, pero puede sugerirse que la cuestión es otra. De fondo, cuando la entrada en la sexualidad activa de las mujeres se ha des-institucionalizado y se configura en la vida de mujer joven un periodo biográfico de una sexualidad autonomizada de la conyugalidad y de la reproducción, a través de una nueva norma, esta vez, de las edades del sexo, introduciría una regulación que al menos lo reduciría. Para las instituciones normativas religiosas no es posible dirigirse hoy la sociedad y la política pública para proponer una normatividad propia de un contexto tradicional (la virginidad) sin el riesgo de no ajustar 
con tendencias culturales que atraviesan a las generaciones ${ }^{26}$. Por cierto, la desinstitucionalización de la primera pareja sexual que se ha producido en las mujeres (ninguna mujer lo hace hoy dentro del matrimonio), expresa una creciente autonomización normativa de los individuos respecto de las instituciones religiosas (Palma, 2009), y la construcción de una legitimidad, más en general, de una sexualidad autonomizada respecto de la conyugalidad y de la reproducción.

En las sociedades desarrolladas contemporáneas el progresivo descenso en las edades del sexo ha sido interpretado como una individualización de las trayectorias sexuales, en el contexto de modificaciones históricas en la estructuración social de un conjunto más amplio de las edades y de reconfiguración de las trayectorias biográficas, de una transformación institucional y normativa de la sexualidad, y al mismo tiempo, como un indicador de transformaciones culturales y sociales más amplias -muy fundamentalmente en las relaciones de género. En la sociedad chilena la transformación de dichas trayectorias ha implicado fundamentalmente a las mujeres situadas en los grupos de altos ingresos, aun cuando con frecuencia se lo invisibiliza. Ellas viven un periodo en el que ésta se encuentra casi enteramente autonomizada tanto de la conyugalidad, como de la reproducción. Dichas mujeres expresan una tendencia cultural según la cual la sexualidad autonomizada ajusta con trayectorias biográficas más individualizadas; y esto a su vez, sugiero observarlo como un asunto vinculado a una apertura a la autonomía sexual de las mujeres. Sugiero analíticamente interrogarse localmente por la autonomía sexual de las mujeres también como un asunto vinculado a la transformación de las edades del sexo.

Antes que el riesgo, es justamente la generación de condiciones para operar dicha separación uno de los elementos fundamentales que da sentido a la demanda feminista por acceso universal a la tecnología preventiva.

Notas
1. Sobre el concepto de medicalización de la sexualidad, véase: Giami, 2000; Tiefer,
1996.
2. Del mismo modo que la estimación de la estructura etaria de la fecundidad (iA 
qué edades tienen hijos/as las mujeres en una sociedad determinada?) se hace al término del periodo reproductivo (49 años), la medición de la edad de la primera relación sexual habría que hacerla con cohortes etarias en las cuales es presumible que una alta proporción de sujetos haya emitido la conducta (haya tenido sexo).

3. Su población son hombres y mujeres entre 18 y 69 años (nacidos entre 1929 y 1980), su muestra son 5.407 sujetos y tiene representatividad nacional.

4. Su población son hombres y mujeres entre 15 y 29 años y ha transitado desde una población de áreas urbanas (1994 y 1997), luego a la representatividad nacional (2000), y finalmente, a la representatividad nacional y regional (desde 2003). La segunda versión (1997) considera 3.446 sujetos, la tercera versión (2000), 7.189 sujetos, la cuarta versión (2003) 6.345 sujetos, la quinta versión (2006), 7.189 sujetos y la sexta versión (2009), 7.570 sujetos. Sus bases de datos están disponibles a partir de la segunda versión en 1997, lo que permite trabajar sobre la población nacida entre 1968 y 1994.

5. Su población son hombres y mujeres de 15 años y más, su muestra son 5.434 sujetos, y tiene representatividad nacional, regional y por zona rural/urbana. http:// www.minsal.gob.cl/portal/docs/page/minsalcl/g_home/submenu_portada_2011/ ens2010.pdf

6. Su población son hombres y mujeres de 15 años y más, su muestra son 6.210 sujetos, y tiene representatividad nacional, regional y por zona rural/urbana http://epi. minsal.cl/estudios-y-encuestas-poblacionales/encuestas-poblacionales/encuestanacional-de-calidad-de-vida-y-salud-encavi/);

7. En la primera etapa se encuestaron 8.131 sujetos y en la segunda etapa, 5874 . Esta Encuesta solo puede ser usada en el estudio de las edades de la primera relación sexual mediante planes de análisis basados en proporciones. http://epi.minsal.cl/ epi/html/invest/EMSE/INFORME\%20EMSE\%202004.pdf

8. Que entendemos genéricamente como uniones, marital o cohabitación.

9. También generación es una categoría analítica que tiene un uso relacionado con las relaciones intergeneracionales familiares, la posición al interior de la estructura de parentesco, asociada a la organización social del ciclo de vida. (Moraes, 2009). La serie de encuestas nacionales de juventud y La Encuesta Nacional de Calidad de Vida no permiten estudiar reconfiguraciones de las trayectorias al interior de la estructura de parentesco, de padres a hijos. Operan con la categoría de jefe de hogar y no usa el de padre o madre, tampoco incorporan en sus cuestionarios preguntas sobre el origen, destinadas a indagar sobre el padre y la madre, su nivel educacional, edad de primer hijo, edad de unión. La importancia de esta perspectiva puede reconocérsela mediante la encuesta GRAVAD, realizada en Brasil, (Heilborn et al, 2006). 10. Esto se vincula con su lógica familiar a su ideología familista. En 1979, la sitúa en una lógica geopolítica, vinculada a la ideología de la seguridad nacional, y se orienta hacia una política pronatalista. Al mismo tiempo, introduce el principio de subsidiariedad, que va introduciendo en el sector salud, en el marco de la transformación del conjunto del Estado. Por ello, la práctica anticonceptiva es conducida al ámbito de lo privado.

11. Es excepcional en este sentido la encuesta sobre trayectorias sexuales realizada en 2005 en el marco del Proyecto del Fondo Global de Lucha contra el SIDA, la tuberculosis y la malaria (Palma et al., 2007).

12. Casadas, solteras cohabitantes, separadas de hecho, viudas, divorciadas, anu- 
ladas.

13. Prefiero utilizar el nivel educacional al nivel socioeconómico al de nivel socioeconómico. Este permite en las generaciones jóvenes poner en relación las trayectorias sexuales, reproductivas y conyugales con las trayectorias educacionales. Esto, porque constituyen un elemento fundamental en la actualidad para comprender dichas trayectorias tanto en su diferenciación social y de género, como en las orientaciones culturales de fondo en las cuales se inscriben. Además, cuando se trabaja con generaciones antiguas y nuevas al mismo tiempo, midiendo eventos que en las primeras son también muy antiguos, es muy probable que el estrato social del pasado no sea similar al actual, por tanto no sean comparables, pues una encuesta consigna el nivel socioeconómico actual. Aun cuando no resuelve enteramente esa limitación, permite una mejor medición porque en las generaciones antiguas la educación tenía una mayor proximidad con la clase social, y una mejor comparación entre las generaciones.

14. El primero de los artículos que se usan como fuente no fue encontrado, sin embargo un resumen del mismo indica que no es un estudio de comportamiento sexual, sino que hace referencia a otros estudios FUENTES M. E, LOBOS L. Adolescente embarazada. Santiago de Chile: Ed. Universitaria, 146, 1994. http://www. bcn.cl/bibliodigital/pbcn/bibliografias/estudios_pdf_bibliografias/EMBARAZOADOLESCENTE.pdf

15. Es una encuesta de salud reproductiva, realizada en 1988, analiza comportamiento sexual y uso de anticonceptivos entre los adultos jóvenes en Chile, con 865 mujeres y 800 hombres de 15-24 años en Santiago. (Valenzuela M. S, Herold J. M., Morris L., López M. [Reproductive health survey of young adults in Greater Santiago]. Bol Oficina Sanit Panam, 1992; 112: 19-30).

16. Quinta Encuesta Nacional de la Juventud, realizada en 2006.

17. Tiene un universo de adolescentes de ambos sexos, entre 12 y 19 años y su muestra son 4.971 consultantes en el Centro de Medicina Reproductiva y Desarrollo Integral de la Adolescencia, de la Facultad de Medicina de la Universidad de Chile entre los años 1990 y 2005, es decir, con sujetos nacidos en un extenso periodo (entre 1971 y 1993).

18. Primero, la cuarta Encuesta Nacional de Juventud, 2003. Segundo, un estudio que trabaja con 198 adolescentes de una comuna de la Región Metropolitana, que corresponden al 14,2\% de los consultantes en consejería en sexualidad de un centro de salud para la atención de adolescentes entre 2000 y 2003, y usa fichas clínicas CLAP/OPS (González et al., 2005).

19. Este estudio fue realizado en 1996-97, es publicado en 2000.

20. http://www.aprendiendoaquerer.cl/vidayfamilia/detalle/id/6

21. http://www.clam.org.br/noticias-clam/conteudo.asp?cod=1357

22. No se indica la fuente y fecha, aunque puede suponerse que sea la versión del año 2003 de la Encuesta Nacional de la Juventud.

23. http://www.humanas.cl/boletin2008/2_trimestre/pdf/proyecto_derechos_sexuales_reproductivos_5933.pdf

24. http://www.teenstar.org/

25. http://www.ucsc.cl/aprendiendo-a-querer/

26. Recién terminada la dictadura, el Centro de Estudios Públicos en un estudio de opinión pública pregunta si es moralmente aceptable que los jóvenes tengan rela- 
ciones sexuales, siempre y cuando haya amor, y 63,7\% responde que es aceptable. Veinte años después ninguna encuesta lo pregunta en el país. http://www.cepchile. cl/dms/lang_1/doc_2931.html

Bibliografía

Bajos N., Guillaume A., Kontula O. Etudes démographiques, no 42, Septembre 2004. Le comportement des jeunes Européens face à la santé génésique. Editions du Conseil de l'Europe.

Bozon M. 1998. "La Sexualité a-t-elle changé?" En: Bajos N; Bozon M; Ferrand A; Giami A; Spira A et le Groupe ACSF. La Sexualité aux Temps du SIDA. Sociologie d aujourd hui. Presses Universitaires de France (PUF). Paris.

Bozon M. "Las encuestas cuantitativas en comportamientos sexuales: emprendimientos sociales y políticos, productos culturales, instrumentos científicos. Sexualidad, Salud y Sociedad". Revista Latinoamericana. ISSN 1984-6487 / n.3 - 2009 - pp.154-170 / www.sexualidadsaludysociedad.org "À quel âge les femmes et les hommes commencent-ils leur vie sexuelle ? Comparaisons mondiales et évolutions récentes". Population E Sociétés. Numéro 391. Juin 2003.

Bozon M, Hertrich V. "Rapports de genre et initiation sexuelle en Afrique et en Amérique latine. Une comparaison à partir de 20 enquêtes EDS", communication au Colloque International "Genre, population et développement en Afrique", Abidjan, juillet 2001.

Bozon M; Kontula O. Initiation sexuelle et genre: comparaison des évolutions de douze pays européens. Population, Année 1997, Volume 52, Numéro 6, $1367-1400$.

De Irala J, Osorio A, Carlos S, Ruiz-Canela M, López-Del Burgo C. Mean age of first sex: Do they know what we mean? Arch Sex Behav 2011. DOI 10.1007/ s10508-011-9779-4

Espinoza O, González L y Uribe D. (2009). Movilidad Social en Chile: El Caso del Gran Santiago Urbano. Revista de Ciencias Sociales (RCS) .Vol. XV, No. 4, Octubre - Diciembre 2009, 586-606.

Fassin E. (2005) LInversion de la Question Homosexuelle. Editions Amsterdam

Fernández L, Bustos L, González L, Palma D, Villagrán J, Muñoz S. “Creencias, actitudes y conocimientos en educación sexual". Revista Médica de Chile 2000; 128:574-583.

Fétis G, Bustos L, Lanas F, Baeza B, Contreras J, Hebel E, Marucich C. “Factores asociados al uso de anticonceptivos en estudiantes de enseñanza media de la comuna de Temuco". REV CHIL OBSTET GINECOL 2008; 73(6):362 -369 .

Gagnon J. 1991. "The Explicit and Implicit Use of the Scripting Perspective in Sex Research, en Gagnon, John, An Interpretation of Desire: Essays in the Study of Sexuality". Series: (WD-CSSGC) Worlds of Desire: The Chicago Series on Sexuality, Gender, and Culture.

Giami A. "Médicalisation de la société et médicalisation de la sexualité ». In : A. Jardin, P. Queneau \& F. Giuliano (eds). Progrès thérapeutiques : la médicali- 
sation de la sexualité en question. Paris: John Libbey Eurotext. 2000. 121-130.

González E., Molina T., Montero A., Martínez V., Leyton C. “Comportamientos sexuales y diferencias de género en adolescentes usuarios de un sistema público de salud universitario". Revista Médica de Chile. 2007; 135: 12611269.

González M., Aguirre I. et al. "Caracterización de asistentes a consejería en sexualidad en un centro de salud integral de adolescentes". Rev. chil. Pediatr.2005; 7 6: 573-579.

Heilborn M., Aquino E., Bozon M., Riva Knauth D. O aprendizado da sexualidade. Reproduçâo e trajetorias socials de jovens brasileiros. Editora Fiocruz; Editora Garamond. Rio de Janeiro, Brasil. 2006.

León P., Minassian M., Borgoño R., Bustamante F. “Embarazo adolescente". Revista Pediatría Electrónica. 2008, Vol 5, Nº 1.

Manheim K. 1990, Le problème des générations 1ère édition allemande: 1928.

Moraes A. Fronteiras da relação. Gênero, geração e a construção de relações afetivas e sexuais Sexualidad, Salud y Sociedad Revista Latinoamericana ISSN 1984-6487 / n.3 - 2009 - pp.10-32

Mossuz-Lavau J.1991 Les lois de l'amour. Les politiques de la sexualité en France (19501990), Paris, Payot.

Palma I., Matus C., Canales M., Palma S., Astorga M. Trayectorias sexuales, vínculos y contextos en la generación que hace su entrada en la sexualidad activa en democracia. Colección Ediciones Conasida-Ministerio de Salud. 2007.

Palma I. "Las instituciones religiosas en la transformación normativa contemporánea en la sexualidad en Chile." Revista de Psicología. Universidad de Chile. Año 2, N³-4. 2009. 11-18.

Palma I. Tesis doctoral: Sociedad chilena en cambio, sexualidades en transformación. 2006. Universidad de Chile. http://www.cybertesis.cl/tesis/uchile/2006/ palma_i/html/index-frames.html

Palma I. Las nuevas generaciones de mujeres, el embarazo no previsto, las edades y la segmentación social en la sociedad chilena. Rev Med Chile 2012; 140: 319-325

Tiefer L. The medicalization of sexuality: Conceptual, normative and professional issues. Annual Review of Sex Research. 1996, 7, 252-282.

Vigil P, Riquelme R, Rivadeneira R, Aranda W. Teenstar: Una opción de madurez y libertad. Programa de educación integral de la sexualidad, orientado a adolescentes Rev Méd Chile 2005; 133: 1173-1182.

Vigil P., Molina C., Encalada M., Cortés E. "La sexualidad de las jóvenes chilenas". ARS MEDICA N 18 (2009): 195-208. 